\title{
Pathogenicity of selected isolates of the quarantine pinewood nematode Bursaphelenchus xylophilus to Scots pine (Pinus sylvestris L.)
}

\author{
Anna Filipiak*
}

Department of Biological Pest Control, Institute of Plant Protection - National Research Institute, Władysława Węgorka 20, 60-318 Poznań, Poland

Received: June 9, 2015

Accepted: October 23, 2015

\begin{abstract}
The pinewood nematode (PWN), Bursaphelenchus xylophilus, is the causal agent of pine wilt disease (PWD). This nematode is considered to be an indigenous to North America and was introduced to Japan in the late 19th century. Subsequently, it has spread throughout Japan and in many other countries, China, Taiwan, and South Korea. In 1999, B. xylophilus was discovered in Portugal, and in 2008 in Spain. So far the studies have revealed that the pathogenicity of B. xylophilus varies between different isolates. The conducted study compared the pathogenicity of five isolates of B. xylophilus, originating from different parts of Japan, to 3-year-old Pinus sylvestris, and their ability to reproduce in the seedlings. The results revealed diverse virulence of $B$. xylophilus resulting in plant mortality. Three isolates S10, Ka4, and T4 caused 100\% mortality of plants within three months while at the same time, the other two isolates, C14-5 and OKD-1 did not cause any disease symptoms on plants. After seven months, some dieback occurred on two seedlings, but similar symptoms were also found on the control plant. Moreover, a significant positive correlation was found between nematode virulence and the number of nematodes reproducing on pine seedlings.
\end{abstract}

Key words: Bursaphelenchus xylophilus, pathogenicity, pinewood nematode, Pinus sylvestris

\section{Introduction}

The pinewood nematode (PWN), Bursaphelenchus $x y$ lophilus, is the causative agent of pine wilt disease (PWD) (Kiyohara and Tokushige 1971). This nematode is considered to be a native species to North America and it was introduced to Japan in the late 19th century (Dropkin et al. 1981; Rutherford et al. 1990; Furuno et al. 1993). After 1980, it spread to other Asian countries (China, Taiwan, and South Korea). Despite the phytosanitary measures provided by the European and Mediterranean Plant Protection Organization (EPPO) and the European Union (EU) to prevent the accidental introduction of B. xylophilus with wood products imported from infested areas, B. xylophilus was detected in Portugal in 1999, and subsequently, in Spain in 2008 (Mota et al. 1999; Filipiak 2008; Robertson et al. 2011). There is a risk of further spread of this pest to other countries in Europe, including Poland. In Poland, pine trees occupy $60 \%$ of the country's area (GUS 2012). The spread of this pest additionally increases due to the presence of at least five Monochamus spp. (M. galloprovincialis, M. sartor, M. sutor, M. saltuarius, and $M$. urussovi). These species are natural vectors of B. xylophilus in other countries (Kozłowski 2003). In Japan, the annual loss of pines caused by pine wilt, reached a maximum value of 2,430,000 $\mathrm{m}^{3}$ in 1979 (Togashi and Shigesada 2006). Nowadays, pine wilt disease annually kills 2,000,000 $\mathrm{m}^{3}$ of pine trees worldwide (EPPO 2015). In Portugal, up till now, B. xylophilus has destroyed more than 1,000,000 ha of pine forests (Robertson et al. 2011). The pinewood nematode is recognised worldwide as one of the most important pests in the forestry industry and is listed as a major plant quarantine objective for most countries in the world (Li 2008).

So far, studies have revealed that the pathogenicity of B. xylophilus associated with conifers, varies between different isolates (Mota et al. 2006). The nematode populations isolated from different coniferous host plants and/or from different regions have been demonstrated as having different virulence on Pinus spp. Virulence ranges from 0 to $100 \%$. However, the virulence of nematode isolates was found not to vary within a single pine or vector insect (Kiyohara and Bolla 1990; Jiao et al. 1996). Virulence of nematode isolates is generally evaluated by the mortality of pine seedlings (mainly more than one year old) inoculated with nematodes (Aikawa and Kikuchi 2007). However, until now, naturally occurring avirulent isolates have been isolated only in Japan (Kiyohara and Bolla 1990).

The main objectives of the study were to compare the pathogenicity of five isolates of B. xylophilus, originating from different parts of Japan, to 3-year-old Pinus sylvestris L. plants, and to compare the ability of these isolates to reproduce in the seedlings. 


\section{Materials and Methods}

In this study, five isolates of B. xylophilus originating from Japan, were examined: C14-5, Ka4, OKD-1, S10, and T4. The isolates were kindly supplied by Dr Yuko Takeuchi from Kyoto University, Japan (the Main Inspectorate of Plant Health and Seed Inspection gave permission to import them for research purposes). The isolates C14-5 and OKD-1 are well known avirulent isolates originating from Japan, which previously have been extensively studied by Takemoto et al. (2005). Prior to examination, all isolates were reared on Botryotinia fuckeliana/malt agar (4.5\%) at $25^{\circ} \mathrm{C}$ for ca 2 weeks. Propagated nematodes were collected by the Baermann funnel method and counted under a stereomicroscope.

The pathogenicity of isolates was assessed in relation to 3-year-old P. sylvestris potted at least six weeks before inoculation (pot height: $13 \mathrm{~cm}$, diameter: $17 \mathrm{~cm}$ ). The substrate contained a mixture of garden turf and sand $(3: 1)$. The experiment began in March when the new growth appeared on the plants. The nematodes were introduced onto plants using the standard method of artificial inoculation (Braasch 2000; Tomalak 2004). A 1-cm long slit was cut on the stem of the young plants, just below the new shoot. A small cotton strip was inserted into the slit. Then, $100 \mu \mathrm{l}$ of water containing 2,500 nematodes were dripped into this slit. The inoculation point was wrapped with a Parafilm strip. Fifty plants were inoculated with five different nematode isolates, i.e. 10 plants per isolate, and 10 control plants were inoculated with water only. The plants were kept in a climatic chamber [lighting $12 \mathrm{~h}$ per day, constant temperature $-25^{\circ} \mathrm{C}$, relative humidity $(\mathrm{RH})-60 \%$ ]. Each plant was irrigated with water twice a week. The plants were observed for symptoms once a week. The first obvious external symptom is the yellowing and wilting of the needles leading to eventual death of the tree, although the whole tree may later show symptoms. Dead plants were cut immediately above the ground. The twigs and needles were removed and the stems were chopped and extracted by the Baermann funnel technique for $24 \mathrm{~h}$ to get the nematodes. Plants that had not totally died off were cut, chopped, and extracted after ten months. The extracted nematodes were counted under a stereomicroscope. The number of nematodes was then determined for the wilted and healthy seedlings.

Results were analysed statistically using analysis of variance (ANOVA). The significance of differences was tested using Tukey's multiple comparison test (significance level 0.05). To achieve homogeneity of variance, a root transformation was used for the number of nematodes. The analysis of regression was performed to examine the cause and effect relationship between the number of days, after which plant death occurred, and the number of recovered nematodes. Statistical analyses were performed using Statistica software (version 10).

\section{Results}

The experiment conducted under controlled conditions revealed varied ability of $B$. xylophilus to cause plant mortality. The first wilting symptoms were observed during the first month of the experiment (Fig. 1). After 4 weeks, isolate $\mathrm{Ka} 4$ caused $60 \%$ plant mortality, isolate $\mathrm{S} 10-40 \%$ plant mortality, and isolate $\mathrm{T} 4-30 \%$ plant mortality. Further plant wilting was observed over the next month. The first complete wilting case was caused by isolate T4, and was observed in the second month of the experiment. At the same time, the other isolates: S10 and Ka4, caused an $80 \%$ mortality of the plants. There was a big increase in the number of wilted P. sylvestris seedlings in the third month of the experiment. The experiment also showed that $100 \%$ of the P. sylvestris seedlings inoculated with isolates S10 and Ka4 wilted in the twelfth week of the experiment.

During the sixth month of the experiment, the two other isolates, C14-5 and OKD-1, did not cause any symptoms of disease on the plants. Two seedlings (one for each of the isolates) showed some signs of dying after seven months, but similar symptoms were also found on a control seedling. The control plants showed a $10 \%$ mortality of the seedlings, but no nematodes were recovered from extracted wood.

In all of the dying plants, characteristic disease symptoms caused by B. xylophilus were observed, i.e. blockage of a sap flow, sudden discoloration of needles, and finally death of the plant.

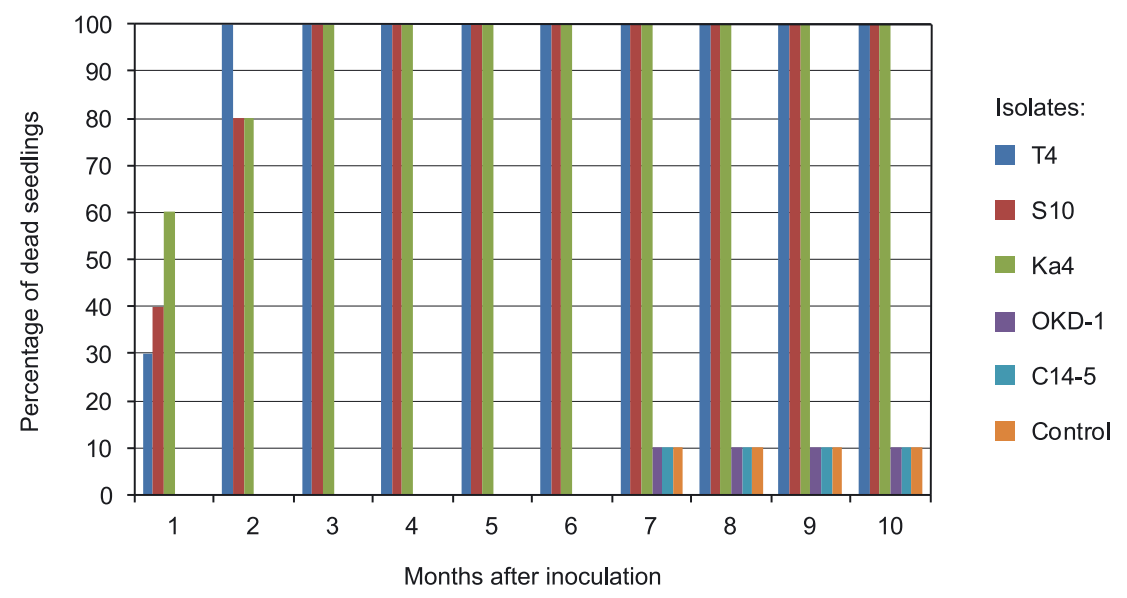

Fig. 1. Mortality of plants caused by tested isolates of Bursaphelenchus xylophilus in subsequent consecutive months of the growing season 
Table 1. Pathogenicity test results of Bursaphelenchus xylophilus on 3-year-old seedlings of Pinus sylvestris under greenhouse conditions and results of grouping based on Tukey's test for average number of re-isolated nematodes per seedling $(\alpha=0.05$; MS $=543.32$; $\mathrm{df}=45)$ and for average number of days after which plant death occurred $(\alpha=0.05$; residual: $\mathrm{MS}=751.80 ; \mathrm{df}=45)$

\begin{tabular}{cccc}
\hline Isolate & $\begin{array}{c}\text { Mortality } \\
{[\%]}\end{array}$ & $\begin{array}{c}\text { Average no. of re-isolated nematodes } \\
\text { per seedling }\end{array}$ & $\begin{array}{c}\text { Average no. of days after which plant } \\
\text { death occurred }\end{array}$ \\
\hline Ka4 & 100 & $11,051.0 \mathrm{~b}$ & $46.3 \mathrm{a}$ \\
$\mathrm{T} 4$ & 100 & $10,660.0 \mathrm{~b}$ & $44.7 \mathrm{a}$ \\
$\mathrm{S} 10$ & 100 & $9,392.0 \mathrm{~b}$ & $52.5 \mathrm{a}$ \\
$\mathrm{C} 14-5$ & 10 & $2,958.5 \mathrm{a}$ & $295.7 \mathrm{~b}$ \\
OKD-1 & 10 & $2,082.0 \mathrm{a}$ & $294.7 \mathrm{~b}$ \\
\hline
\end{tabular}

Means followed by the same letters are not significantly different

After the death of the plants or at the final end of the experiment, the numbers of re-isolated nematodes were counted (Table 1). The number of nematodes re-isolated from the seedlings ranged from 40 to over 21,000 specimens. The average number of nematodes re-isolated was the highest in the P. sylvestris seedling inoculated with isolate Ka4 (Fig. 2). The lowest average number of nematodes was recovered from the seedling inoculated with isolate OKD-1. The highest number of nematodes, amounting to 21,000, was recovered from a single seedling inoculated with isolate T4. The average number of nematodes re-isolated was higher in dead seedlings than in healthy seedlings. The average number of nematodes re-isolated from wilted seedlings inoculated with all the five isolates was almost identical, and a similar trend was observed among the healthy seedlings. The highest number of nematodes recovered from a healthy seedling was 3,400 from a plant inoculated with isolate C14-5. A significant positive correlation was shown between nematode virulence and the number of nematodes reproduced on pine seedlings.

The analysis of variance was conducted for a number of days after which plant death occurred and for the total number of recovered nematodes (a root of the number of nematodes based on a root transformation). The analysis of variance revealed significant differences between the tested combinations (respectively: $\mathrm{F}_{1}=244.3$; $\mathrm{df}=4,45$; $\left.\mathrm{p}<0.001 ; \mathrm{F}_{2}=15.5 ; \mathrm{df}=4,45 ; \mathrm{p}<0.001\right)$. Isolates $\mathrm{T} 4, \mathrm{Ka} 4$, and S10 were significantly different from isolates C14-5 and OKD-1 and formed two homogeneous groups (Table 1).

The results of the analysis of variance for regression showed a highly significant effect of the number of days after which plant death occurred on the number of recovered nematodes $(\mathrm{F}=54.9 ; \mathrm{df}=4.8 ; \mathrm{p}<0.001)$ (Fig. 3$)$.

\section{Discussion}

Previous studies demonstrated that the virulence level of B. xylophilus varies greatly among isolates and that there are differences in the reactions of pine trees to infection by virulent and avirulent B. xylophilus isolates (Ikeda and Suzuki 1984; Kiyohara and Bolla 1990; Aikawa and Kikuchi 2007). Until now, non-pathogenic isolates of B. xylophilus have been confirmed only from Japan, while naturally occurring avirulent isolates of B. xylophilus have not yet been isolated in the United States or in Europe. Virulence varies greatly among isolates of this nematode from pines within the same stand and different stands in Japan. These differences could arise because of host or geographic isolation. The differences may also have been influenced by environmental stress, including the range of seasonal temperatures (Rutherford and Webster 1987; Kiyohara and Bolla 1990).

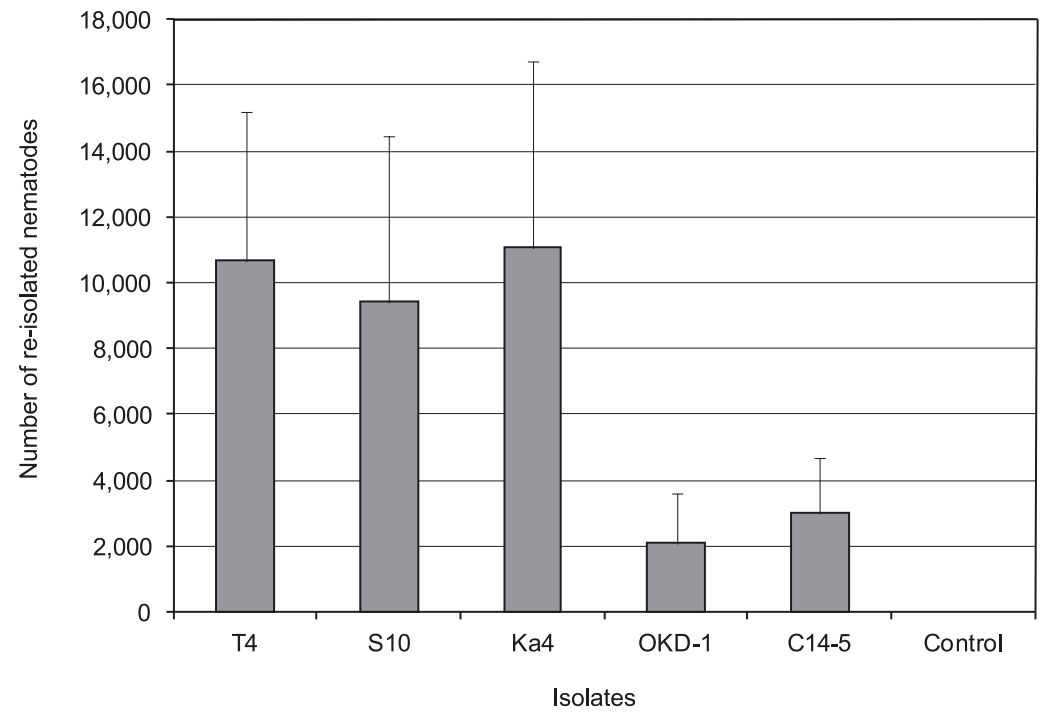

Fig. 2. The ability to reproduce different Bursaphelenchus xylophilus isolates in inoculated seedlings (mean \pm SD) 


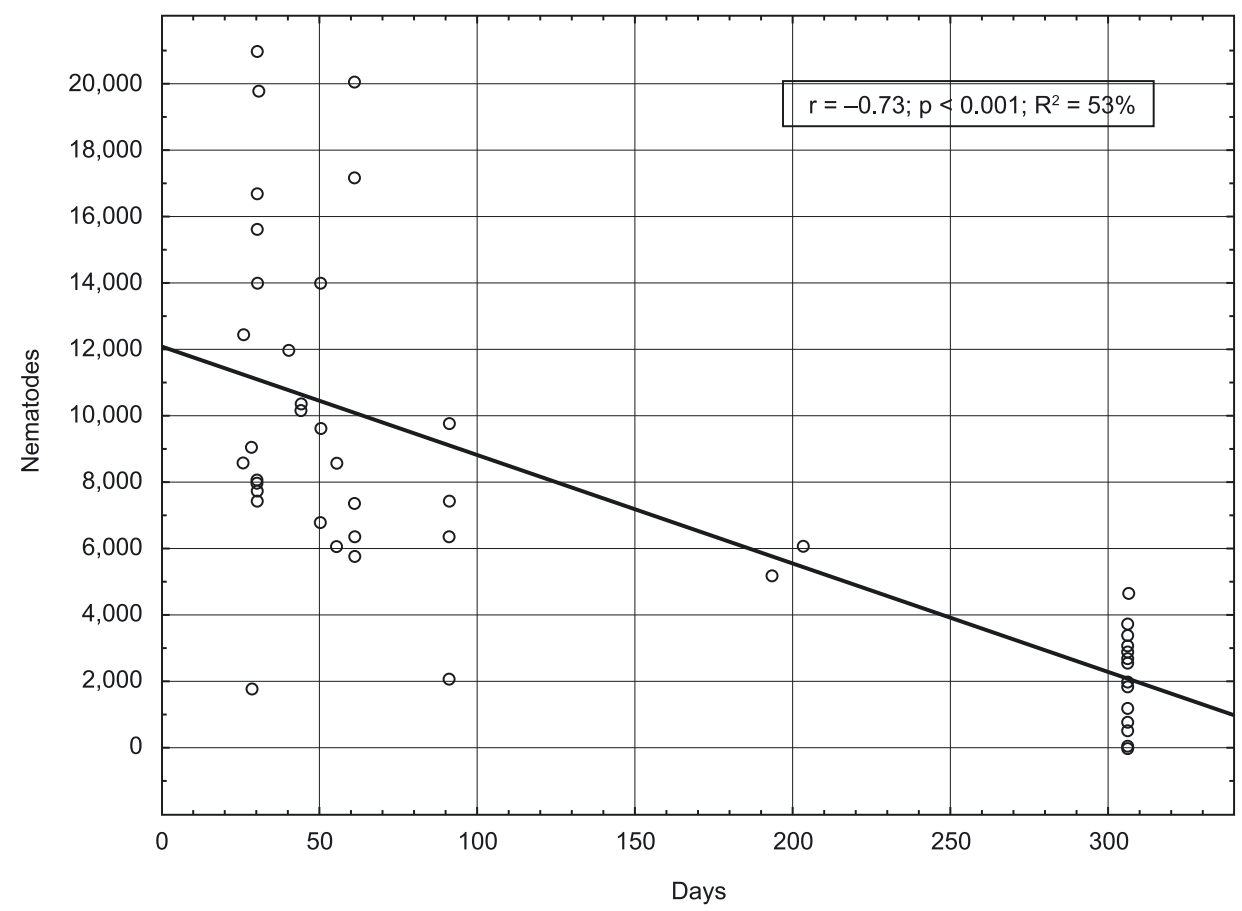

Fig. 3. A scatterplot with a fitted regression line (the number of re-isolated nematodes with respect to the changing number of days)

In this study, the nematode virulence and reproduction were investigated using $P$. sylvestris. The species, P. sylvestris, is one of the most susceptible species to B. $x y$ lophilus, and it is the most widely spread conifer species in central and northern Europe (Futai and Furuno 1979; Caroppo et al. 2000). The conducted study confirmed the different levels of pathogenicity of B. xylophilus to P. sylvestris seedlings. The examined isolates $\mathrm{Ka} 4, \mathrm{~S} 10$, and $\mathrm{T} 4$ were highly pathogenic and caused a $100 \%$ mortality of plants during the three months of the experiment, while the C14-5 and OKD-1 isolates seemed to be nonpathogenic to $P$. sylvestris seedlings. During the study only one wilted seedling was observed for each of these isolates.

To date, several studies have reported on the reproductive ability of B. xylophilus isolates, with different levels of virulence, under in vitro and in vivo conditions. The studies indicated a significant positive correlation between virulence and the reproductive ability of B. xylophilus on the B. fuckeliana culture (Aikawa and Kikuchi 2007). Virulent isolates reproduced four times as abundantly as avirulent ones when cultured on fungal mats on B. fuckeliana at $25^{\circ} \mathrm{C}$ for 6 days (Kiyohara and Bolla 1990; Wang et al. 2005; Aikawa and Kikuchi 2007). In the in vivo situation, when virulent and avirulent isolates separately inoculated $P$. thunbergii seedlings, nematode density in the case of the virulent isolates, increased with time after inoculation (Aikawa et al. 2006; Aikawa and Kikuchi 2007).

In this study, reproductive ability was examined using B. xylophilus isolates with different virulence. A highly positive correlation was found between virulence and reproductive ability of B. xylophilus. For the Ka4, S10, and T4 isolates, causing $100 \%$ mortality of plants during the three month period, significantly higher numbers of reisolated nematodes were recorded when compared to the C14-5 and OKD-1 isolates. Moreover, the healthy seedlings inoculated with the C14-5 and OKD-1 isolates had a significantly lower number of nematodes when compared to the wilted seedlings. This suggests that nematode virulence is closely associated with its reproductive ability, irrespective of the in vitro or in vivo conditions.

However, nematode virulence cannot be explained only by its reproductive ability. The ability of nematodes to establish in pine tissues, and to feed on living cells in pine tissues, are also thought to be important factors for determining the virulence level. Nematode infection of a host tree is impossible without these attributes (Aikawa and Kikuchi 2007). It was mentioned, that composition and/or activity of the enzymes related to the degradation of the cell wall in pine tissues is one of the factors determining the virulence level of nematodes (Kojima et al. 1994). Moreover, some studies suggest that the secreted proteins are essential molecules for parasitism by B. $x y$ lophilus (Shinya et al. 2013; Espada et al. 2015).

This study compared the pathogenicity of different B. xylophilus isolates originating from Japan. So far, the occurrence of isolates of this nematode with varying degrees of pathogenicity has been confirmed from that region, only. In future research, it may be interesting to compare the pathogenicity of B. xylophilus isolates originating from Europe and North America and to elucidate the relationship between virulence and reproductive ability of the nematode using pine species other than $P$. sylvestris.

\section{Acknowledgements}

The author sincerely thanks Dr Yuko Takeuchi from Kyoto University, Japan, for providing live cultures of B. xylophilus isolates. Special thanks also to Prof. Maria Kozłowska, from the Poznan University of Life Sciences, for her help and advice on statistical analysis. The author is greatly indebted to Ms. Agnieszka Błaszak for her dedicated technical assistance in the laboratory work. 
The research was financially supported by the Polish Ministry of Agriculture and Rural Development, through the Long-term Programme of the Institute of Plant Protection - National Research Institute, Poznań, Poland, Project 2.1: "Identification of areas potentially threatened by the invasion of the quarantine pine wilt nematode, Bursaphelenchus xylophilus, and the elaboration of an optimal strategy for control of this pest."

\section{References}

Aikawa T., Kikuchi T., Kosaka H. 2006. Population structure of Bursaphelenchus xylophilus within single Pinus thunbergii trees inoculated with two nematode isolates. Forest Pathology 36 (1): 1-13.

Aikawa T., Kikuchi T. 2007. Estimation of virulence of Bursaphelenchus xylophilus (Nematoda: Aphelenchoididae) based on its reproductive ability. Nematology 9 (3): 371-377.

Braasch H. 2000. Influence of temperature and water supply on mortality of 3-year-old pines inoculated with Bursaphelenchus xylophilus and B. mucronatus. Nachrichtenblatt des Deutschen Pflanzenschutzdienstes 52 (10): 244-249.

Caroppo S., Cavalli M., Coniglio D., Ambrogioni L. 2000. Pathogenicity studies with various Bursaphelenchus populations on conifer seedlings under controlled and open air conditions. Redia 83: 61-75.

Dropkin V.H., Foudin A., Kondo E., Linit M., Smith M., Robbins K. 1981. Pinewood nematode: a threat to U.S. forests? Plant Disease 65: 1022-1027.

EPPO 2015. Prepared by CABI and EPPO for the EU under Contract 90/399003. Available on: http://www.cabi.org/isc/datasheet/10448 [Accessed: April 22, 2015]

Espada M., Silva A.C., Akker S.E., Cock P.J.A., Mota M., Jones J.T. 2015. Identification and characterization of parasitism genes from the pinewood nematode Bursaphelenchus $x y-$ lophilus reveals a multilayered detoxification strategy. Molecular Plant Pathology. DOI: 10.1111/mpp.12280

Filipiak A. 2008. The pine wilt disease. Sylwan 12: 9-19.

Furuno T., Nakai I., Uenaka K., Haya K. 1993. Pine wilt disease of introduced pine species planted in Kamigamo and Shirahama Experimental Stations of Kyoto University Forest - resistance of Pinus spp. to the pine wood nematode. The Reports of the Kyoto University Forests 25: 20-34. (in Japanese)

Futai K., Furuno T. 1979. The variety of resistances among pine species to pine wood nematode, Bursaphelenchus lignicolous. Bulletin of the Kyoto University Forests 51: 23-26.

GUS - Główny Urząd Statystyczny 2012. Leśnictwo. Informacje i opracowania statystyczne. Warszawa, 341 ss.

Ikeda T., Suzuki T. 1984. Influence of pine-wood nematodes on hydraulic conductivity and water status in Pinus thunbergii. Journal of the Japanese Forestry Society 66 (10): 412-420. (in Japanese, with English summary)

Jiao G., Shen P., Li H. 1996. A study on the pathogenicity of pine wood nematodes from Japan and Nanjing, China to Cedrus deodara and Pinus massoniana. Plant Quarantine 10 (4): 193195. (in Chinese, with English summary)

Kiyohara T., Tokushige Y. 1971. Inoculation experiments of a nematode, Bursaphelenchus sp., onto pine trees. Journal of the Japanese Forestry Society 53: 210-218. (in Japanese, with English summary)
Kiyohara T., Bolla R.I. 1990. Pathogenic variability among populations of the pinewood nematode, Bursaphelenchus xylophilus. Forest Science 36 (4): 1061-1076.

Kojima K., Kamijyo A., Masumori M., Sasaki S. 1994. Cellulase activities of pine-wood nematode Bursaphelenchus xylophilus isolates with different virulences. Journal of the Japanese Forestry Society 76: 258-262. (in Japanese, with English summary)

Kozłowski M.W. 2003. Native and exotic sawyer beetles, Monochamus spp. (Coleoptera, Cerambycidae) as the vectors of the pine wilt nematode, Bursaphelenchus xylophilus. Sylwan 1: 24-34.

Li H. 2008. Identification and pathogenicity of Bursaphelenchus species (Nematoda: Parasitaphelenchidae). Ph.D. thesis, Ghent University, Ghent, Belgium, 223 pp.

Mota M.M., Braasch H., Bravo M.A., Penas A.C., Burgermeister W., Metge K., Sousa E. 1999. First report of Bursaphelenchus xylophilus in Portugal and in Europe. Nematology 1 (7-8): 727-734.

Mota M., Takemoto S., Takeuchi Y., Hara N., Futai K. 2006. Comparative studies between Portuguese and Japanese isolates of the pinewood nematode, Bursaphelenchus xylophilus. Journal of Nematology 38 (4): 429-433.

Robertson L., Cobacho Arcos S., Escuer M., Santiago Merino R., Esparrago G., Abelleira A., Navas A. 2011. Incidence of the pinewood nematode Bursaphelenchus xylophilus Steiner \& Buhrer, 1934 (Nickle, 1970) in Spain. Nematology 13 (6): 755-757.

Rutherford T.A., Webster J.M. 1987. Distribution of pine wilt disease with respect to temperature in North America, Japan and Europe. Canadian Journal of Forest Research 17 (9): 1050-1059.

Rutherford T.A., Mamiya Y., Webster J.M. 1990. Nematode-induced pine wilt disease: factors influencing its occurrence and distribution. Forest Science 36 (1): 145-155.

Shinya R., Morisaka H., Kikuchi T., Takeuchi Y., Ueda M., Futai K. 2013. Secretome analysis of the pine wood nematode Bursaphelenchus xylophilus reveals the tangled roots of parasitism and its potential for molecular mimicry. PLoS One 8 (6): e67377.

Takemoto S., Kanzaki N., Futai K. 2005. PCR-RFLP image analysis: a practical method for estimating isolate-specific allele frequency in a population consisting of two different strains of the pinewood nematode, Bursaphelenchus xylophilus (Aphelenchida: Aphelencoididae). Applied Entomology and Zoology (Jpn) 40: 529-535.

Togashi K., Shigesada N. 2006. Spread of the pinewood nematode vectored by the Japanese pine sawyer: modeling and analytical approaches. Population Ecolology 48 (4): 271-283.

Tomalak M. 2004. Ocena przydatności dostępnych metod do identyfikacji taksonomicznej kwarantannowego nicienia węgorka sosnowca [Bursaphelenchus xylophilus (Steiner et Bührer) Nickle]. [Evaluation of methods available for taxonomic identification of the Bursaphelenchus xylophilus]. Progress in Plant Protection/Postępy w Ochronie Roślin 44 (1): 452-461.

Wang Y., Yamada T., Sakaue D., Suzuki K. 2005. Variations on the life history parameters and their influence on rate of population increase of different pathogenic isolates of the pine wood nematode, Bursaphelenchus xylophilus. Nematology 7 (3): 459-467. 